\title{
Advantages of Three-Dimensional Space-Time Frames
}

\author{
Tower Chen ${ }^{1, *}$, Zeon Chen ${ }^{2}$ \\ ${ }^{1}$ Unit of Mathematical Sciences, College of Natural and Applied Sciences, University of Guam, UOG Station, Mangilao, Guam 96923, \\ USA \\ ${ }^{2}$ Independent Researcher, CA, USA \\ zeon_chen@yahoo.com, tchen@uguam.uog.edu
}

\begin{abstract}
Time dilation and length contraction formulas are derived in Special Relativity. However, these phenomena cannot be visualized in four-dimensional space-time frames. From the time dilation formula, we can derive a relationship between space and time for a pair of inertial frames and, in turn, construct three-dimensional space-time frames based on this relationship. Furthermore, length contraction can be shown on these frames. Since space and time are inseparable, neither time dilation nor length contraction can be fully understood unless discussed together. We apply three-dimensional space-time frames to describe the motion of an object in the twin paradox. Using the concept of the center of mass of a pair of inertial frames, we are able to determine who is on the rest frame of a pair of inertial frames. This example demonstrates the advantage of three-dimensional space-time frames over four-dimensional space-time frames in describing relativistic motion.
\end{abstract}

Keywords Time Dilation and Length Contraction, Twin Paradox, A Pair of Inertial Frames, Center of Mass, Three-Dimensional Space-Time Frames, Four-Dimensional Space-Time Frames

\section{Introduction}

In classical Newtonian physics, space and time are absolute quantities. Hence, space and time can be treated independently and discussed separately. With his Theory of Relativity, Einstein proved that space and time are dependent and must not be treated separately. However, in four-dimensional space-time frames (4-D S-T frames), space and time are treated independently. Thus, we seek a frame in which space and time are dependent. From the time dilation formula, we derive a relationship between space and time for a pair of inertial frames[1] and, in turn, construct three-dimensional space-time frames (3-D S-T frames) based on this relationship. Furthermore, length contraction can be illustrated on these frames. Thus, 3-D $\mathrm{S}-\mathrm{T}$ frames facilitate the joint discussion and comprehension of time dilation and length contraction, as neither concept can be fully understood without the other.

In the twin paradox, the elder twin takes a journey on a space shuttle to a distant star and returns to Earth. Using 4-D S-T frames, it is hard to visualize that the elder twin will actually look younger than his younger twin upon his return. From the concept of the center of mass of a pair of inertial frames, we are able to determine that the younger twin is on the rest frame and the elder twin is on the moving

* Corresponding author:

tower_c@yahoo.com (Tower Chen)

Published online at http://journal.sapub.org/fs

Copyright (C) 2012 Scientific \& Academic Publishing. All Rights Reserved frame. We will employ 3-D S-T frames to describe the space shuttle's motion as observed from both frames.

\section{Relationship of Space and Time Between a Pair of Inertial Frames}

The time dilation formula

$$
t=\frac{t^{\prime}}{\sqrt{1-(u / c)^{2}}}
$$

and the length contraction formula

$$
x^{\prime}=x \sqrt{1-(u / c)^{2}}
$$

from Special Relativity are well accepted. In the formulas, c represents the speed of light, $u$ represents the relative velocity between a pair of inertial frames, $t$ and $t^{\prime}$ represent regular (dilated) time and proper (original) time, and $x$ and $x^{\prime}$ represent proper (original) and regular (contracted) length[2].

\subsection{Time Dilation}

Multiplying both sides of the time dilation formula (1) by $c$ and squaring both sides, we arrive at

$$
(c t)^{2}=\frac{\left(c t^{\prime}\right)^{2}}{1-(u / c)^{2}}
$$

Equation (3) can be further simplified to

$$
(c t)^{2}-(u t)^{2}=\left(c t^{\prime}\right)^{2}
$$

Letting $r=c t, x=u t$, and $h=c t^{\prime}$, we obtain the following relationship

$$
r^{2}-x^{2}=h^{2}
$$


This relationship between space and time for a pair of inertial frames cannot be illustrated with Cartesian coordinate frames, where the t-axis has the same scale for both frames. However, the relationship in equation (5) can be illustrated with 3-D S-T frames, which utilize polar coordinates.

Figure 1 illustrates a thought experiment scenario, which will be referenced throughout this paper. A train moves with constant velocity $\mathrm{u}$ with respect to the station platform. At time $t=0$, the origin $O^{\prime}$ of the train's coordinate frame coincides with origin $O$ of the platform's coordinate frame, and at that moment a photon is emitted vertically with velocity $c$ by an observer on the train.

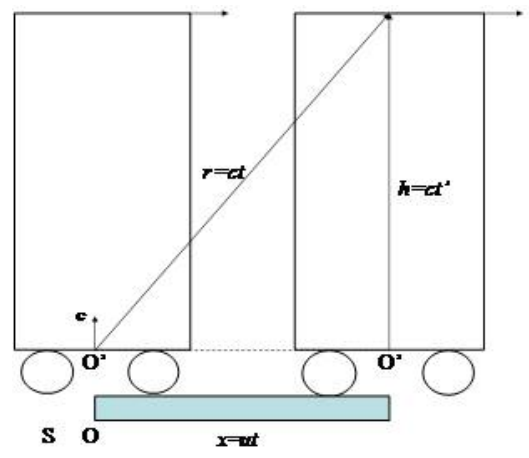

Figure 1. A train moves with constant velocity u with respect to the station platform. At time $t=0$, the origin O' of the train's coordinate frame coincides with origin $\mathrm{O}$ of the platform's coordinate frame, and at that moment a photon is emitted vertically with velocity $\mathrm{c}$ by an observer on the train

If it takes time $t$ ' for the photon to hit the train's ceiling of height $h$, as measured by the observer on the train, then

$$
t^{\prime}=h / c
$$

To the observer on the platform, the photon travels a distance $r$ in the time $t$ it takes for the photon to reach the ceiling, hence

$$
t=r / c
$$

which must be longer than $t^{\prime}(6)$. The distance that the train has traveled in that time $t$, as measured by the observer on the platform, is

$$
x=u t
$$

The distance that the train has traveled in time $t^{\prime}$, as measured from the observer on the train is

$$
x^{\prime}=u t^{\prime}
$$

The location of the photon on the $x-y$ plane, as measured by the observer on the train at time $t^{\prime}$ is

$$
h=c t^{\prime}
$$

The location of the photon on the $x-y$ plane, as measured by the observer on the platform at time $t$ is

$$
r=c t
$$

\subsubsection{4-D S-T Frame}

In classical physics, in order to describe the motion of an object along the $x$-axis with time, the time axis is placed perpendicular to the $x$-axis, which treats space and time as independent. It is possible to convert the units of time for the time axis into units of distance by multiplying time with the velocity of a medium selected for keeping track of time.
In Figure 2, a train $O^{\prime}$ moves with constant velocity $u$ with respect to the station platform $O$, and a ball is thrown vertically with a velocity $v$ with respect to the train, at the moment the origins of the frames coincide. We can construct a Cartesian coordinate system to describe the ball's motion on the pair of inertial frames: one set on the train and the other on the station platform.

We can convert the unit for the time axis $t(\mathrm{sec})$ into units of distance $(m)$ by taking the product of $v$ and $t$. With this set-up, line $O^{\prime} A$ describes the motion of the train along $x$-axis with time $t$ and also represents the actual distance that the ball travels within the time interval $t$ on the $x-y$ plane with respect to the observer on the train. Additionally, line $O A$ describes the motion of the train along the $x$-axis with time $t$ and also represents the actual distance that the ball travels within the time interval $t$ on the $x-y$ plane with respect to the observer on the platform.

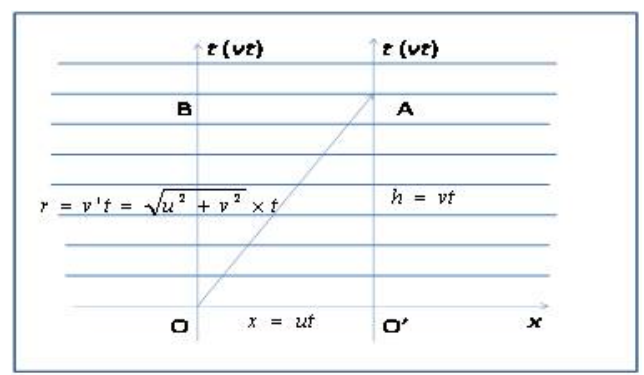

Figure 2. A train moves with constant velocity $u$ with respect to the station platform. At time $t=0$, the train's frame origin $O$ ' coincides with the platform's frame origin $O$, and a ball is thrown vertically with velocity $v$ with respect to the train. A Cartesian coordinate system describes the ball's motion on the pair of inertial frames: train and platform. The unit for the time axis $t(\mathrm{sec})$ is converted into units of distance $(\mathrm{m})$ by taking the product of $v$ and $t$

Because of $O A$, the distance that the ball travels within the time interval $t$ with respect to the observer on the platform, is equal to

$$
r=\sqrt{(v t)^{2}+(u t)^{2}}
$$

the velocity of the ball with respect to the observer on the platform is

$$
v^{\prime}=r / t=\sqrt{(v t)^{2}+(u t)^{2}} / t=\sqrt{v^{2}+u^{2}}
$$

This velocity satisfies the relationship between space and time, $r^{2}-x^{2}=h^{2}$ (5), which was established earlier from the time dilation formula, where $r=v^{\prime} t=\sqrt{v^{2}+u^{2}} \times t, x=u t$, and $h=v t$.

Now, instead of throwing a ball straight upwards, the observer on the train tosses a photon instead. We set about the same exercise of creating a Cartesian coordinate system to describe the motion of an object on the pair of inertial frames: one set on the train and the other one set on the station platform. We again convert the units of the time scale $\mathrm{t}$ to units of distance by taking the product of $t$ and $c$, replacing the speed of the ball with the speed of the photon. With this set-up, line $O A$ can be used to describe the motion of the train along the $x$-axis, but line $O A$ cannot represent the actual distance that the photon travels within the time interval $t$ on the $x-y$ plane with respect to the observer on the 
platform, unless we let $c^{\prime}=\sqrt{c^{2}+u^{2}}$. This means that in order to preserve the relationship between space and time derived from the time dilation formula, $r^{2}-x^{2}=h^{2}$ (5), we must let $c^{\prime}=\sqrt{c^{2}+u^{2}}$. This is in direct contradiction with one of Einstein's postulates for Special Relativity, which states that the speed of light is always $c$ regardless of the frame of reference.

\subsubsection{3-D S-T Frame}

The Michelson-Morley experiment demonstrated that the speed of light is constant regardless of the frame of reference. Hence, the velocity of the emitted photon, as measured from the observer on the platform, is

$$
c^{\prime}=c,\left(c^{\prime} \neq \sqrt{c^{2}+u^{2}}\right)
$$

In Figure 3, we have the same scenario discussed earlier, where a train $O^{\prime}$ moves with constant velocity u with respect to the station platform $O$, and a photon thrown straight upwards with a velocity $c$ with respect to the train. Rather than representing this using 4-D S-T Cartesian coordinates we do so using 3-D S-T frames, which utilize polar coordinates, to describe an object's motion along the $x$-axis from the perspective of the observer on the station platform and the observer on the train. In 3-D S-T frames, time is represented by concentric circles of varying radii about the origins of the frames, instead of lines that run parallel to the $x$-axis in the case of 4-D S-T Cartesian frames. The units of time for the time circles can be converted to units of distance by taking the product of $c$ and $t$. If the unit of time is seconds, then the radii of the circles have units of light-seconds; if the unit of time is years, then the radii of the circles have units of light-years. The radii of the circles centered about origin $O$ represent time $t$ relative to the platform and have lengths equal to $c t$. The radii of the circles centered origin $O$, represent time $t$ ' relative to the train and have lengths equal to $c t^{\prime}$. With this set-up, line $O A$ describes the train's motion along the $x$-axis and also represents the motion of the photon on the $x-y$ plane as observed from the platform; line $O^{\prime} A$ describes the train's motion and also represents the motion of the photon on the $x-y$ plane as observed from the train; and $O O^{\prime}$ is the distance of the train from the platform along the $x$-axis.

With the 3-D S-T frames, the relationship between space and time that was derived from the time dilation formula $r^{2}-x^{2}=h^{2}(5)$ is preserved, and the speed of light remains $\mathrm{c}$ regardless of the frame of reference. It is worth noting that when the velocity of the train $u<<c$, i.e. we are dealing with speeds in the realm of classical physics, the 3-D S-T frames will approximate the time-to-distance modified Cartesian frames discussed in Figure 2.

\subsection{Length Contraction}

We can derive the length contraction equation $x^{\prime}=x \sqrt{1-(u / c)^{2}}$ (2) by multiplying both sides of the time dilation equation (1) by $u$. This equation shows that the distance, as measured from the train $x$, is shorter than the distance, as measured from the platform $x$, since

$$
t^{\prime} \leq t
$$

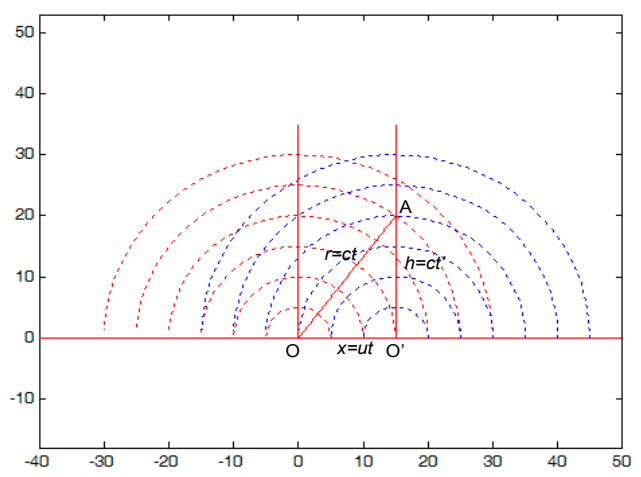

Figure 3. 3-D S-T frames are used to describe the same scenario discussed earlier: a train moves with constant velocity $\mathrm{u}$ with respect to the station platform. At time $t=0$, the origin $O$ ' of the train's coordinate frame coincides with origin $O$ of the platform's coordinate frame, and at that moment a photon is emitted vertically with velocity $c$ by an observer on the train. Using 3-D S-T frames, the relationship between space and time for a pair of inertial frames, $r^{2}-x^{2}=h^{2}$, is preserved while maintaining the invariance of the speed of light

Length contraction is illustrated in Figure 4. In this thought experiment, we have the same scenario discussed earlier, where a train $O^{\prime}$ moves with a constant velocity u with respect to the station platform $O$, and a photon is thrown straight upwards with a velocity $c$ with respect to the train when the origins of the frames coincide. To measure the train's location, $x$, multiple sensors are installed along the railroad tracks, and one sensor is installed on the train. When the train's senor contacts a sensor on the railroad tracks, time is clocked as $\mathrm{t}$ from the platform and as $\mathrm{t}$ ' from the train. The train's location at time $\mathrm{t}$ is $x=u t(8)$, with respect to the platform, and its location at time $t^{\prime}$ is $x^{\prime}=u t^{\prime}(9)$, with respect to the train.

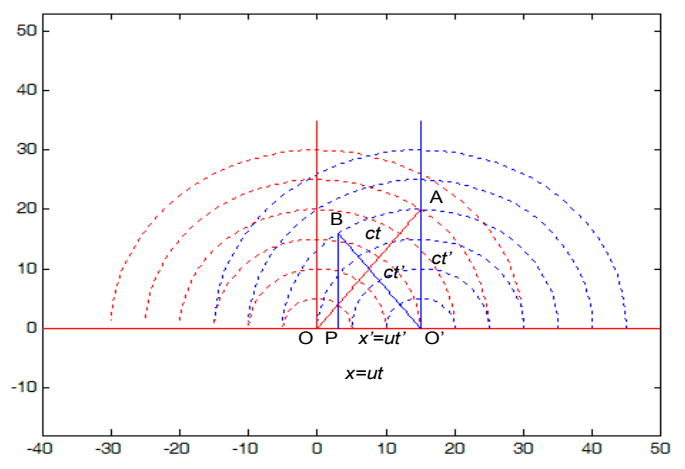

Figure 4. 3-D S-T frames are used to describe the same scenario discussed earlier: a train moves with constant velocity u with respect to the station platform. At time $t=0$, the origin $O$ ' of the train's coordinate frame coincides with origin $O$ of the platform's coordinate frame, and at that moment a photon is emitted vertically with velocity $\mathrm{c}$ by an observer on the train. Length contraction experienced by the observer on the moving frame can be visualized using these 3-D S-T frames

In Figure 4, Line $O A$ represents the train's location with respect to the platform, and line $O^{\prime} B$ represents the platform's location with respect to the train. The lines 
illustrate that an object ( $O O^{\prime}$ ) laid on the rest frame will be shorter when measured by the moving frame $\left(O^{\prime} P\right)$. Since

$$
\frac{u}{c}=\frac{u}{c} \Rightarrow \frac{u t}{c t}=\frac{u t^{\prime}}{c t^{\prime}} \Rightarrow \frac{x}{r}=\frac{x^{\prime}}{r^{\prime}}
$$

The last relationship shows that triangle $O A O^{\prime}$ is similar to triangle $O^{\prime} B P$.

\section{Determining a Rest Frame}

In the twin paradox, it is hard to accept that the elder of a pair twins, who travels to a distant star and returns will look younger than their younger sibling, who remains on Earth. This is because the younger twin can just as easily say that, relative to the space shuttle, the earth receded and then made its way back to the space shuttle. To resolve the twin paradox, determining which twin is actually moving is important.

If a pair of inertial frames exists in isolation, observers on either frame could consider themselves to be on the rest frame. From a kinetic viewpoint, there is no real rest frame for these two frames. From a dynamic viewpoint, the total momentum of the two frames in an isolated system does not change in the absence of external forces, because momentum is conserved. This also means that in the absence of external forces, the center of mass for the system, i.e. the pair of inertial frames, does not move. Thus, for an isolated system not subjected to external forces, the reference frame set from the perspective of the center of mass is the rest frame.

In the twin paradox scenario, the earth, the space shuttle, and the gases expelled by the shuttle's boosters together comprise the system of interest. The center of mass of this system is

$$
\vec{R}=\frac{M \vec{r}_{1}+m_{S} \vec{r}_{2}+m_{G} \vec{r}_{3}}{\left(M+m_{S}+m_{G}\right)}
$$

where $M$ is the mass of the earth, $m_{S}$ is the mass of the shuttle, and $m_{G}$ is the mass of the gas. Since the earth's mass is much larger than that of the shuttle and the gas $\left(M>>m_{S}\right.$ and $M>>m_{G}$ ), the earth gains almost zero acceleration from $F=m a$ between the earth and the shuttle and

$$
\vec{R} \approx \vec{r}_{1}
$$

This means that the center of mass for this system, for all intents and purposes, coincides with that of the earth. Because the reference frame set from the perspective of the center of mass is the rest frame, in the twin paradox scenario, we can consider the reference frame of the younger twin, who remains on earth, to be the rest frame.

\section{Application}

From the discussion in the previous section, we established that, in the twin paradox, the earth should be treated as the rest frame which means that the space shuttle should be treated as the moving frame. Indeed, it is the space shuttle that actually travels to the distant star and back and not a journey that is made by the earth. The following discussion explains why the elder twin on the moving frame should look younger than the younger twin on the rest frame.

The elder twin visits a star 15 light-years $(c \times y r)$ away from Earth on a space shuttle. The shuttle reaches $60 \%$ of the speed of light immediately after launch and travels the remaining distance to the star at that speed. The younger twin on Earth measures the outbound trip to the star to take 25 years, where

$$
t=\frac{15(c \times y r)}{0.6 c}=25 y r
$$

After reaching the star, the elder twin returns to Earth. The shuttle reaches $80 \%$ of the speed of light immediately after launch and travels the remaining distance to Earth at that speed. The younger twin on Earth measures the inbound trip to Earth to take 18.75 years, where

$$
t=\frac{15(c \times y r)}{0.8 c}=18.75 y r
$$

According to the younger twin on Earth, the entire trip took 43.75 years (25 years to reach the star and 18.75 years to return).

What was the trip's length according to the elder twin? The motion of the space shuttle's round trip is shown in Figure 5 using 3-D S-T frames. The shuttle's outbound trip from earth to the star along line $A B$ is described by line $A C$ from the younger twin's (earth's) perspective and by line $B C$ from the elder twin's (the shuttle's) perspective[3]. The shuttle's inbound trip from the star to earth along line $B A$ is described by line $C D$ from the younger twin's (earth's) perspective and by line CE from on the elder twin's (the shuttle's) perspective[4].

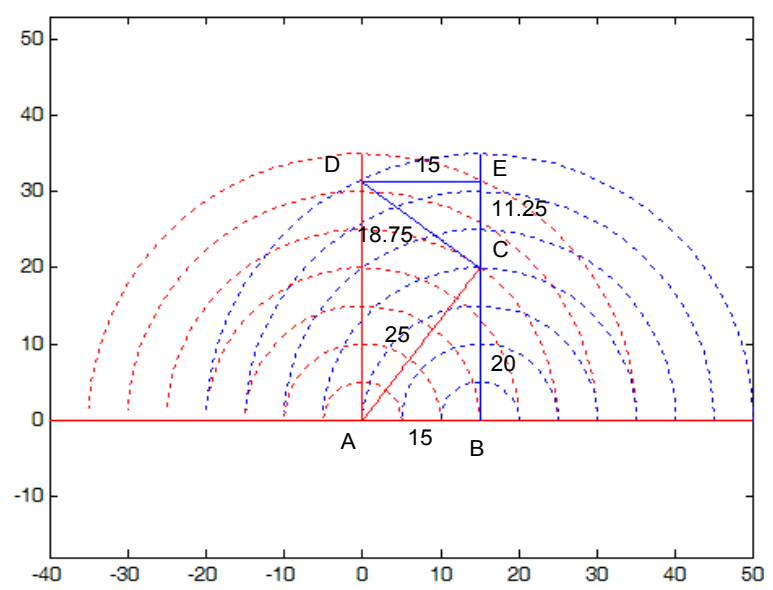

Figure 5. The motion of the space shuttle in the twin paradox scenario, described using 3-D S-T frames. Lines $A C$ and $C D$ represent the shuttle's outbound trip and inbound trips, respectively, as observed by the younger twin on earth. Lines $B C$ and $C E$ represent the shuttle's outbound trip and inbound trip, respectively, as observed by the elder twin on the shuttle

For the outbound trip, the younger twin on earth measures the trip to take 25 years for the space shuttle moving with velocity $0.6 \mathrm{c}$ to travel the distance 15 light-years. However, the elder twin on the space shuttle measures the trip to take 20 years, where

$$
t^{\prime}=(25 y r) \sqrt{1-(0.6 c / c)^{2}}=20 y r
$$


For the inbound trip, the younger twin on earth measures the trip to take 18.75 years for the space shuttle moving with velocity $0.8 \mathrm{c}$ to travel the distance of 15 light-years. On the other hand, the elder twin on the space shuttle measures the trip to take 11.25 years, where

$$
t^{\prime}=(18.75 y r) \sqrt{1-(0.8 c / c)^{2}}=11.25 y r
$$

From the elder twin's perspective, the entire trip takes only 31.25 years to complete ( 20 years to reach the star and 11.25 years to return). Thus, the elder twin should look 12.5 years (the difference of 43.75 years and 31.25 years) younger than the younger twin upon his return.

All calculations are shown in Figure 5, which was created using 3-D S-T frames. For the outbound trip, the distance between Earth and the star is

$$
x=u t=15(c \times y r)
$$

The distance light travelled in 25 years is

$$
r=c t=25(c \times y r)
$$

The distance light travelled in 20 years is

$$
r^{\prime}=h=c t^{\prime}=20(c \times y r)
$$

For the inbound trip, the distance between Earth and the star is (23). The distance light travelled in 18.75 years is

$$
r=c t=18.75(c \times y r)
$$

The distance light travelled 11.25 years is

$$
r^{\prime}=h=c t^{\prime}=11.25(c \times y r)
$$

The round trip distance, as measured by the younger twin, is 30 light-years, the sum of 15 light-years for the outbound trip and 15 light-years for the inbound trip. The round trip distance, as measured by the elder twin, is only 21 light-years, the sum of 12 light-years for the outbound trip and 9 light-years inbound trip. The distance 12 light-years is $\mathrm{x}^{\prime}$ calculated from the previously established relationship in (16), given values $x=15, r=25$, and $r^{\prime}=20$. The distance 9 light-years is $x^{\prime}$ calculated from that same relationship, given values $x=15, r=18.75$, and $r^{\prime}=11.25$. The difference in distances as measured by the twins (9 light-years) is consistent with the elder twin looking (12.5years) younger than the younger twin. The elder twin experiences a shorter distance travelled than what the younger-twin measures and not a slowing of his clock. The dilation in time is accompanied by a corresponding contraction in length; each phenomenon is important in understanding the other. Though the relative velocity of a pair of inertial frames is used in calculating time dilation and length contraction, the experiences of the observers on the different frames will be dependent on who is really making the journey.

Figure 6 uses a 3-D S-T frame to illustrate a space shuttle traveling at different percentages of the speed of light with respect to the earth. As the velocity of space shuttle approaches the speed of light, the line representing the shuttle's motion in space with time approaches the $x$-axis. For the same period of time as measured by the younger twin (in this example 4 circles or 4 light-years), as the speed of the elder twin's spaceship increases, the corresponding time (the vertical lines) measured by the elder twin gets shorter and shorter as the speed of the shuttle approaches c. For the twin paradox scenario discussed earlier, let us consider the extreme case where the space shuttle moves with the velocity of light, $c$.

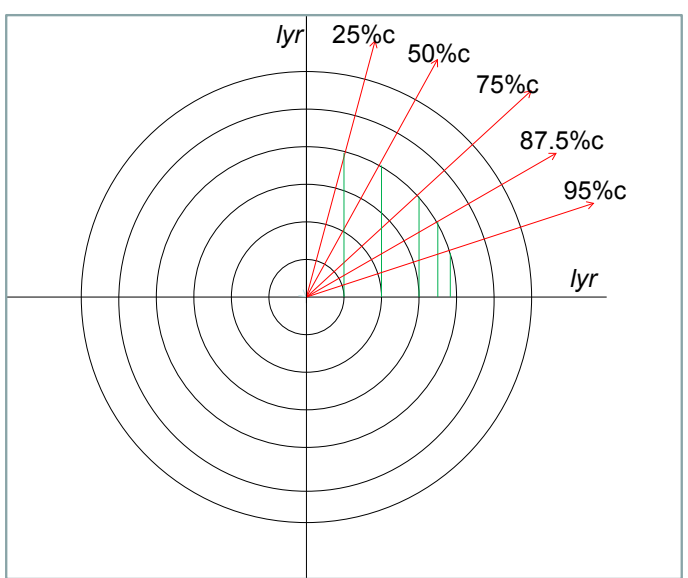

Figure 6. Space shuttles traveling at various percentages of the speed of light are depicted. Lines closer to the vertical axis describe slower shuttles, and lines closer to the horizontal axis describe faster shuttles

For the outbound trip, the younger twin on Earth measures the trip to take 15 years for the space shuttle, moving with velocity $c$, to travel a distance of 15 light-years. However, the elder twin on the space shuttle measures the trip to take 0 years, where

$$
t^{\prime}=(15 y r) \sqrt{1-(c / c)^{2}}=0 y r
$$

For the inbound trip, the younger twin on Earth measures the trip to take 15 years for the space shuttle, moving with the velocity $c$, to travel a distance of 15 light-years. On the other hand, the elder twin on the space shuttle measures the trip also to take 0 years, also shown by (28). For the elder twin, the entire trip takes 0 years $(0$ years to reach the star and 0 years to return). Thus, upon his return, the elder twin should look 30 years (the difference of 30 years and 0 years) younger than the younger twin.

The round trip distance traveled by the space shuttle, as measured by the younger twin is 30 light-years, by summing 15 light-years for the outbound trip and 15 light-years for the inbound trip. The round trip distance, as measured by the elder twin is only 0 light-years, by the summing 0 light-years for the outbound trip and 0 light-years for the inbound trip. 0 light-years is $x^{\prime}$ calculated from the following relationship in (16), given values $x=15, r=15$, and $r^{\prime}=20$ for both outbound and inbound trips. The elder twin on the space shuttle traveling with the velocity of light will experience no time and no distance.

\section{Conclusions}

The relationship of space and time between a pair of inertial frames can be derived from the time dilation formula. 3-D S-T frames are constructed to satisfy and maintain that relationship between space and time and the invariance of the speed of light. These frames aid in the visualization of time dilation and length contraction, which cannot be 
illustrated 4-D S-T frames. In 3-D S-T frames, one important distinction between space and time is that space is bidirectional and time is unidirectional, i.e. an object can proceed forward and backward in space but can only proceed forward in time. In the proposed 3-D S-T frame, the representation of time as concentric spheres of different radii centered about the origin of the spatial axes inherently restricts time to a single direction, i.e. negative time (circles with negative radii) has no meaning.

From a dynamic viewpoint, we can distinguish between the rest frame and moving frame using the center of mass of the pair of inertial frames. With this, we can explain why the elder twin looks younger than the younger twin in the twin paradox. This twin paradox can also be visualized through intuitive, geometric graphs. These benefits certainly demonstrate the value of 3-D S-T frames.

\section{REFERENCES}

[1] T. Chen and Z. Chen, "A Pair of Inertial Frames Versus an Inertial Frame," Concepts of Physics, vol. V, pp. 523-534, 2008.

[2] T. Chen and Z. Chen, "Time Dilation and Length Contraction Shown in Three-Dimensional Space-Time Frames," Concepts of Physics, vol. VI no. 2, 2009.

[3] T. Chen and Z. Chen, "The Paradox of Twins Described in a Three-Dimensional Space-Time Frame," in Proc. 13th Asian Technology Conference in Mathematics; Suan Sunandha Rajabhat University, Thailand; 2008.

[4] T. Chen and Z. Chen, "Motions of Particles Described in a Three-Dimensional Space-Time Frame," in Proc. 10th Asian Technology Conference in Mathematics; Korea National University of Education; 2005. 